\title{
UM NOVO MÉTODO PARA IDENTIFICAÇÃO DE CÂMERA A PARTIR DE IMAGENS DIGITAIS
}

\section{A NEW METHOD FOR SOURCE CAMERA IDENTIFICATION FROM DIGITAL IMAGES}

Guilherme Sekine; Danillo Roberto Pereira; Francisco Assis da Silva; Mário Augusto Pazoti; Helton Molina Sapia

Faculdade de Informática - FIPP, Universidade do Oeste Paulista - Unoeste e-mail: guilherme_sekine@hotmail.com, danilopereira@unoeste.br

RESUMO - A identificação da câmera responsável pela captura de uma dada imagem pode ser utilizada como indícios de vários tipos de crimes. Essa identificação pode ocorrer com o uso de um método científico que possa determinar qual câmera é a geradora de uma determinada imagem. Cada câmera gera imagens com algumas particularidades, devido à características individuais delas. Inserido nesse contexto, este trabalho foi baseado na criação de um novo método de identificação da câmera geradora a partir de imagens, por meio de estudos e implementação de algoritmos de processamento digital de imagens. Uma nova base de dados também foi gerada para realização de treinamentos e testes do método proposto.

Palavras-chave: Computação Forense; Identificação de Câmera.

ABSTRACT - The identification of the camera responsible for the capture of a given image may be used as indicia of various kinds of crimes. This identification can occur with the use of a scientific method that can determine which camera is the generator of a particular image. Each camera generates images with some peculiarities due to their own features. Within this context, this work was based on the creation of a

Recebido em: $15 / 05 / 2015$

Revisado em: 10/10/2015

Aprovado em: 18/11/2015 new generating camera identification method from images, through studies and implementation of digital image processing algorithms. A new database was also generated for conducting training and testing of the proposed method.

Keywords: Computer Forensisc; Camera Identification.

\section{INTRODUÇÃO}

As câmeras digitais estão cada vez de mais fácil acesso para qualquer cidadão, devido ao aumento da tecnologia digital, à diminuição considerável de seu custo. Tal popularização ocasionou um aumento nos crimes que podem ser cometidos por meio dessas câmeras, como por exemplo, pornografia infantil, pirataria e/ou chantagem e etc. Logo, criou-se a necessidade de identificar a fonte geradora (máquina) das imagens usadas nesses crimes e dessa forma "definir" se um suspeito do crime é o dono do dispositivo em questão. Essa análise é similar a um exame de balística; que procura determinar qual a arma a responsável pelo disparo de um dado projétil.

A partir dessa situação, se tornou necessário a criação de um método matemático/estatístico que possibilite indicar, com alto grau de confiabilidade, a fonte geradora de uma dada imagem.

Cada câmera deixa padrões particulares e únicos nas suas imagens geradas, causadas por poeira ou sujeira na sua lente e principalmente por hot/dead pixels nos sensores da câmera (pontos dos sensores que captam "muito" mais ou "muito" menos luz que os demais). Observe os padrões citados na Figura 1. 

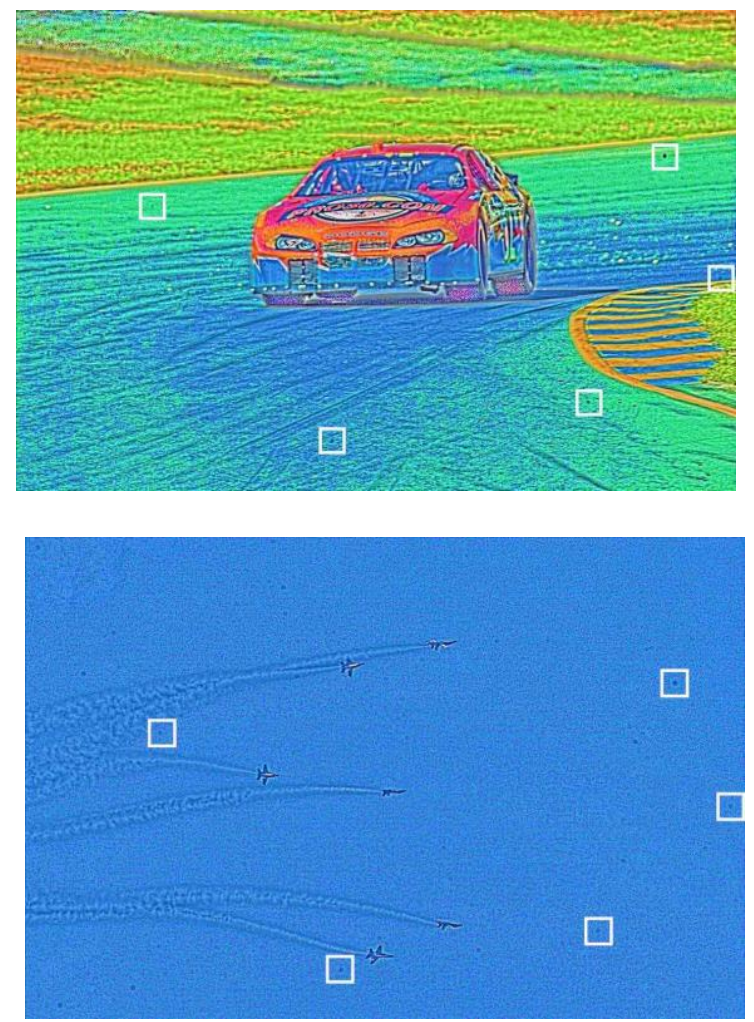

Figura 1. Exemplo de padrões persistentes gerados pela câmera em suas aquisições. Fonte: (DIRIK; SENCAR; NEMON, 2008).

Esses padrões tornam possível relacionar uma imagem a um conjunto de imagens gerada por uma mesma câmera. Para definir a provável fonte geradora de uma imagem é necessário identificar tal padrão em um conjunto de imagens e compará-los com a imagem de entrada.

O objetivo de identificar o autor da aquisição da imagem por meio de um método computacional está diretamente ligado a computação forense. É chamada de computação forense a área de estudo que pesquisa métodos científicos com o objetivo de encontrar soluções para todo o tipo de análise, recuperação, preservação, interpretação e coleta de evidências digitais com intuito de facilitar a tarefa de analisar materiais digitais em busca de provas/indícios para julgamentos. A importância da utilização da computação forense cresce, conforme o crescimento da tecnologia, que de forma geral, acaba por envolver cada vez mais dispositivos digitais em crimes, e consequentemente, gera mais evidências digitais para serem analisadas nesse sentido.

O restante do artigo está organizado da seguinte forma: Na Seção 2 está a
Fundamentação Teórica. A Abordagem Proposta (Descrição do Método, Pseudocódigo, Diferentes Abordagens) é o tema da Seção 3. Na seção 4 estão os resultados de experimentos. A conclusão e os trabalhos futuros encontram-se descritos na Seção 5.

\section{FUNDAMENTAÇÃO TEÓRICA}

$O$ método que provavelmente é o mais simples e prático de descobrir a origem (câmera que gerou a imagem) de uma dada imagem é a utilização do cabeçalho EXIF (Exchangeable Image File Format - Formato de Arquivo de Imagem Intecambiável), que contém algumas informações acessíveis, como por exemplo: data e hora da aquisição da imagem, orientação, resolução, utilização ou não de flash, etc. Toda imagem gerada por uma câmera digital possui além dessas informações o modelo e a marca da câmera geradora da imagem (veja a Figura 2). Contudo esses dados podem ser facilmente alterados ou excluídos, sendo assim, inconfiáveis, haja vista que para ser usado como prova/indício em um tribunal o método deve ter alto grau de confiabilidade/acertos e não ser de fácil adulteração.

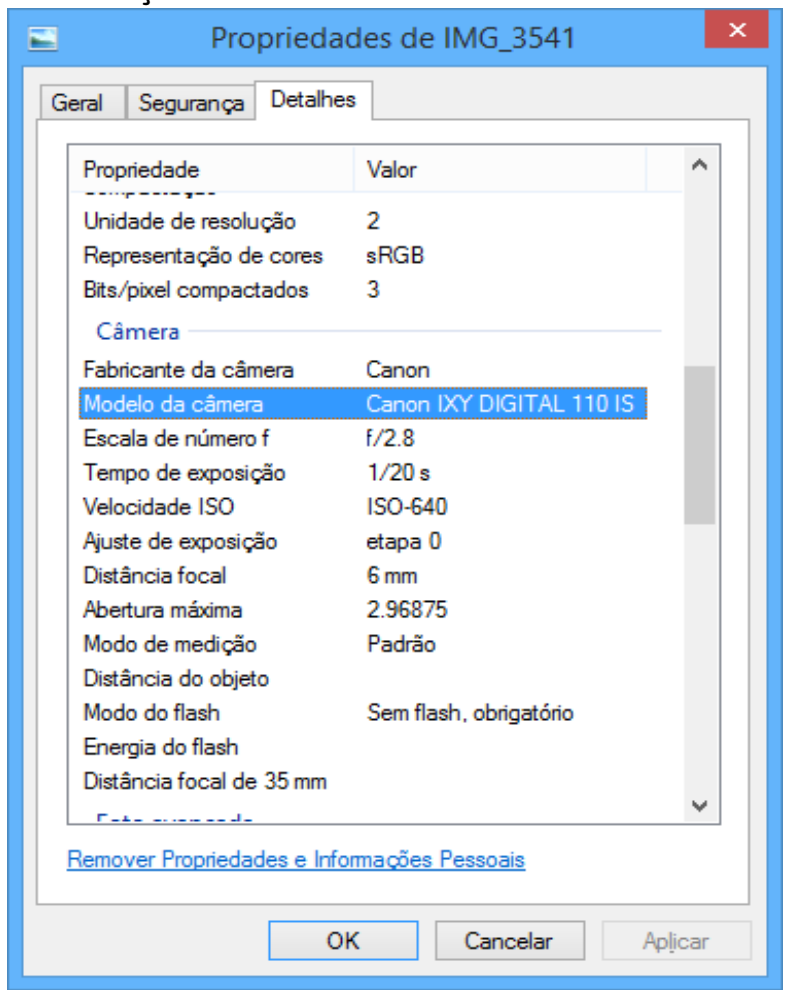

Figura 2. Visualização dos dados do cabeçalho EXIF no sistema operacional Windows 8.

\subsection{TRABALHOS RELACIONADOS}

Nos trabalhos encontrados na literatura, foram notadas várias maneiras diferentes de identificar características deixadas pelas câmeras. 
0 grande desafio comumente reportado foi o fato do conteúdo real da imagem ser capaz de dificultar a percepção desses detalhes, que muitas vezes podem ser altamente relevantes para a definição da câmera geradora.

\subsubsection{Identificação do modelo da câmera}

Segundo Rocha et al. (2011), para a identificação do modelo de aquisição da imagem, são estudadas características de hardwares que compõem a câmera como um todo, como por exemplo: tipo/tamanho dos sensores, informações da lente, tipo de filtro do mosaico/demosaico utilizado e algoritmos utilizados na lógica de processamento da câmera.

Porém, de acordo com Dirik, Sencar e Nemon, (2008), o que desvaloriza a utilização desse tipo de característica é o fato de que muitos modelos de câmeras utilizam componentes de um mesmo fabricante (lente, sensor, etc), o que ocasiona a coincidência de mesmos componentes em modelos diferentes, tornando assim, o processamento das imagens iguais ou muito similares.

Ainda sobre a identificação de modelo de aquisição, Kharrazi, Sencar e Menon (2004), indica uma maneira mais confiável de se obter resultado positivo: a presença de artefatos de cor, que são inseridos na imagem durante $o$ processo de demosaico (obtenção de informações da luminância dos pixels vizinhos para determinar o valor do pixel central). Levando em conta essa informação, os autores apresentam uma proposta com a extração de 34 características, para imagens coloridas, onde obtiveram um bom índice de aproveitamento com diferentes tipos de modelos de dispositivos. Entre as características citadas estão valores médios de pixels, distribuição do centro de massa dos pixels vizinhos, estatísticas do domínio Wavelet e correlação entre pares de banda RGB.

Foram reportados resultados entre $78.7 \%$ e $95.2 \%$ de acerto, considerando cinco modelos de câmeras.

\subsubsection{Imperfeições nos sensores}

Kurosawa, Kuroki e Saitoh (1999) sugere a utilização de imperfeições nos sensores dos dispositivos como o criador de padrões nas imagens.

Os autores acreditam que a identificação do ruído padrão causado por dark currents em câmeras digitais pode ser relevante na caracterização do sensor de captura de uma imagem. Dark current é o que faz com que os elétrons se acumulem em cada pixel devido à ação termal, que é encontrada nas junções inversas dos pinos e independe da luz incidente. Segundo eles, o valor final do pixel está fortemente relacionado a esse fator, que é particular de cada sensor.

Os autores propuseram que detecção de defeitos localizados dos pixels, intensificando os ruídos de padrão da imagem. Porém, essa técnica é bastante limitada, já que câmeras mais sofisticadas possuem sensores especiais (com funcionamento diferente) e mecanismos de hardware para correção automática da imagem no momento de sua aquisição. Não foram reportados resultados obtidos pelo método.

\subsubsection{Poeira nos sensores}

Dirik, Sencar e Nemon (2008) mostra um estudo para identificar a presença de poeira nos sensores das câmeras no momento de aquisição da foto. Os autores acreditam que a presença dessas partículas de poeira nos sensores criam um padrão em todas as imagens que forem criadas por ela, logo é possível identificar a imagem a fonte da imagem.

Esse trabalho obteve uma alta taxa de acerto ( $94 \%$ de acurácia), porém foi utilizado um cenário de apenas duas câmeras e com padrões relativamente fáceis de se identificar, sua base de dados foi disponibilizada pelos autores e inclusive foi usada nos testes relatados na Seção 3 .

A grande falha do estudo baseado em poeira no sensor é o fato de que essa característica é temporal, a poeira no sensor pode ser limpada a qualquer momento, então a partir disso o padrão deixará de existir nas próximas imagens capturadas por ela. Isso seria relevante na área forense.

\subsubsection{Pixels defeituosos}

Com uma abordagem parecida com a anterior, Geradt et al. (2001) acredita que a carga gerada em cada pixel no momento de captura pode levar a criação de um pixel supersaturado (hot pixel) caso a carga seja maior que o normal, ou a um pixel pouco saturado (dead pixel) para aqueles que possuem pouca carga ou nenhuma carga.

Assim como no trabalho de Dirik, Sencar e Nemon (2008), os autores desse afirmam que essas características geram um padrão permanente em todas imagens capturadas pela câmera, tornando possível a identificação da 
fonte geradora da imagem através da identificação desse padrão.

O ponto negativo desse método é o fato de que nem todas câmeras possuem pixels defeituosos e também as câmeras mais sofisticadas possuem sensores especiais que realizam a correção desses defeitos no momento da aquisição da imagem.

O trabalho não apresenta resultados de testes, nem base de dados utilizada para avaliação.

\subsubsection{Extração de ruídos}

As técnicas mais efetivas atualmente analisam os efeitos de ruído inserido no processo de captura das imagens, Lukas e Goljan (2006) abordam uma forma de realizar a estimativa do padrão de ruído dos sensores para encontrar a fonte geradora de uma imagem.

Uma forma utilizada para relacionar uma imagem a uma câmera é extrair o ruído de um conjunto de imagens geradas por uma câmera e realizar o cálculo da média desse conjunto, essa média é o padrão de ruído da câmera em questão e é feita a correlação entre ela e o ruído da imagem em investigação.

Dois tipos de padrões de ruídos foram abordados pelos autores: padrão de ruído fixo (causado pelas dark currents, que foram descritos por um tópico anterior) e ruído decorrente da foto-responsividade não uniforme (Photo Response Non-Uniformity Noise - PRNU). O padrão de ruído fixo é de uso limitado, conforme também foi descrito anteriormente.

Caso o valor de correção ultrapassar um limiar determinado previamente, os autores consideram que a imagem em questão foi obtida pela câmera referente ao padrão utilizado nessa correlação.

Bons resultados foram reportados por esse trabalho, chegando a atingir 95\% de acurácia. Contudo, o trabalho apresentou uma alta taxa de falso-positivos no momento em que foi considerado um cenário de nove câmeras.

0 método descrito até aqui recebeu uma melhoria proposta por Li (2010). O autor afirma que o ruído obtido pela técnica descrita sofre forte influência da cena, com alta frequência nas bordas dos objetos. Ele propõe a utilização de modelos matemáticos para aprimorar o ruído residual, com o objetivo de eliminar ou diminuir a interferência que detalhes da cena causam no resultado.
O resultado foi aumentado para $93,4 \%$ com 15 câmeras no treinamento, porém, o método altera os valores dos pixels, o que pode dificultar a identificação da origem das imagens.

\section{ABORDAGEM PROPOSTA}

Nesta seção serão descritas a criação da base de imagens e a abordagem proposta para o método.

\subsection{CRIAÇÃO DA BASE DE IMAGENS}

A base de dados foi criada com 2 câmera digitais de marcas diferentes (Samsung e Canon), onde cada uma delas capturou 140 imagens diferentes, em ambientes semelhantes entre elas, como mostra a Figura 3. Cada conjunto de imagem será divido, cada câmera terá 85 imagens para realizar o treinamento $\mathrm{Tr}$ e outras 55 para testes Te.

A base foi nomeada GSDP e se encontra disponível no endereço https://www.dropbox.com/sh/mbbnrxtnhee3f25 /AADd22ygzRxT_eK6BYScVOCZa?dl=0
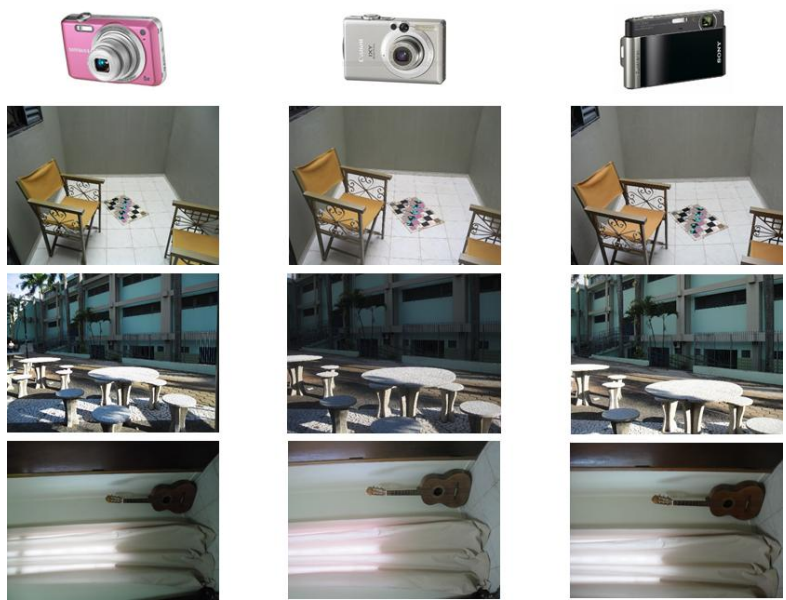

Figura 3. Demonstração da forma que foram capturadas as imagens para o conjunto de câmeras.

\subsection{ABORDAGEM PROPOSTA}

A ferramenta foi desenvolvida na linguagem de programação Java e sua metodologia, chamada de SCZ será descrita a seguir.

Para cada câmera gera-se uma imagem $\mathbf{M}$, que corresponde a "média" de todas as imagens do conjunto Tr. Veja na Figura 4 uma imagem, onde cada pixel é obtido pela soma dos pixels de todas as imagens, divido pela quantidade total delas. Esta etapa de pré-processamento permite destacar na imagem $\mathbf{M}$ o possível padrão que a câmera gera em suas imagens (hot/dead pixels). 


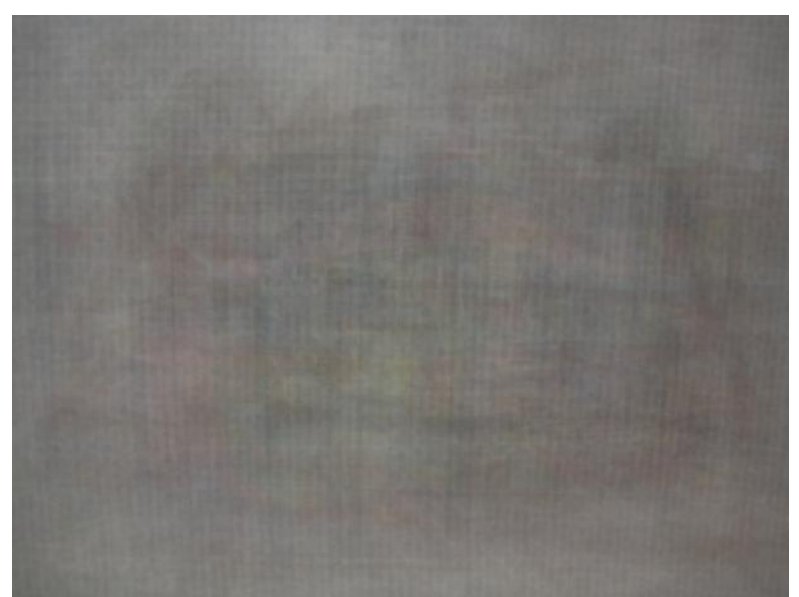

Figura 4. Representação da média da base de treinamento (para câmera Samsung).

Na imagem $\mathbf{M}$, é realizada uma filtragem que identifica se há (para cada canal - vermelho, verde e azul), algum pixel que esteja fora do intervalo $\left[\mathbf{m}-\left(\boldsymbol{\sigma}^{*} \boldsymbol{\alpha}\right), \mathbf{m}+\left(\boldsymbol{\sigma}^{*} \boldsymbol{\alpha}\right)\right.$ ], onde $\mathbf{m}$ é a média dos pixels de uma máscara KxK e $\boldsymbol{\sigma}$ é seu desvio padrão e a é um fator de sensibilidade. Em caso positivo, esse pixel será considerado como o um possível padrão. Por exemplo: se $\mathbf{M}[\mathrm{x}, \mathrm{y}]>\mathbf{>} \mathbf{m}$ $+\left(\sigma^{*} \boldsymbol{\alpha}\right)$ conclui-se que o pixel na coordenada $[x, y]$ é um hot pixel, que será marcado para o passo seguinte. De maneira análoga se se $\mathbf{M}[\mathrm{x}, \mathrm{y}]$ $<=\mathbf{m}-\left(\boldsymbol{\sigma}^{*} \boldsymbol{\alpha}\right)$ esse será um dead pixel.

Esses pontos encontrados geram uma assinatura digital de cada câmera, que contém os pixels que são considerados dead/hot pixels. Nessa imagem é realizado o processo de erosão para eliminar pequenos ruídos na imagem, permanecendo somente os pontos relevantes, como mostrado na Figura 5 a seguir.

Figura 5. Representação da assinatura digital da câmera Samsung, canal vermelho (antes dos processos de erosão/dilatação).

O passo final será a comparação dos pixels em todas as coordenadas identificadas da assinatura digital (os hot/dead pixels de cada câmera), entre a imagem "média" $\mathbf{M}$ e a imagem de entrada.

Foram estudadas diferentes métricas para o passo final, onde o valor a ser considerado pelas comparações pode ser pela média de todas as diferenças dos pixels, pela mediana deles, pela maior diferença obtida entre todas, pela menor diferença e/ou por uma combinação linear entre o valor máximo e o mínimo. Essa variação na forma de realizar o passo final do método, gerou alterações nos resultados e serão apresentados na Seção seguinte.

A câmera que obtiver a menor métrica, levando em consideração os canais RGB, será considerada a câmera responsável pela captura.

A Figura 6 mostra os pixels comparados e seus respectivos canais.

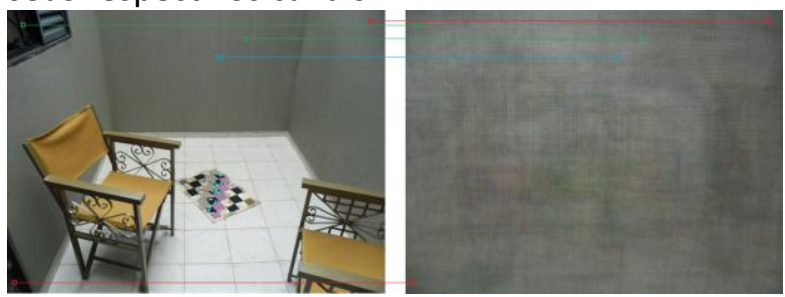

Figura 6. Os canais dos pixels nas coordenadas da assinatura digital são comparados entre a imagem em observação.

\section{EXPERIMENTOS E RESULTADOS}

Nesta seção serão descritos todos os experimentos realizados com a ferramenta e os resultados obtidos em cada base de dados testada.

\subsection{BASE CRIADA}

Na comparação de 2 câmeras de marcas diferentes (Canon e Samsung), da base descrita na seção anterior, foi obtido o resultado de $87,27 \%$ de acertos, em um filtro de valor $\mathbf{K}$ sendo 5. Com um total de 140 imagens de cada dispositivo, sendo 85 para treino e 55 para o teste. Os testes com imagens da câmera Canon tiveram 47 acertos e 8 erros, já os da Samsung indicaram 49 acertos e 6 erros. 
Tabela 1. O resultado de cada tipo de métrica na consideração referente as comparações entre as posições, e com $\mathbf{K}$ valendo 5

\begin{tabular}{|l|c|c|c|c|c|}
\hline Câmera & $\begin{array}{c}\text { Médi } \\
\mathbf{a}\end{array}$ & $\begin{array}{c}\text { Median } \\
\mathbf{a}\end{array}$ & $\begin{array}{c}\text { Maio } \\
\mathbf{r}\end{array}$ & $\begin{array}{c}\text { Meno } \\
\mathbf{r}\end{array}$ & $\mathbf{M}+\mathbf{m}$ \\
\hline $\begin{array}{l}\text { Samsun } \\
\mathrm{g}\end{array}$ & $83,6 \%$ & $70,9 \%$ & $89 \%$ & $70,9 \%$ & $89 \%$ \\
\hline Canon & $83,6 \%$ & $80 \%$ & $\begin{array}{c}81,8 \\
\%\end{array}$ & $78,1 \%$ & $\begin{array}{c}85,4 \\
\%\end{array}$ \\
\hline Média & $83,6 \%$ & $75,4 \%$ & $\begin{array}{c}85,4 \\
\%\end{array}$ & $74,5 \%$ & $\begin{array}{c}87,2 \\
\%\end{array}$ \\
\hline
\end{tabular}

Tabela 2. Mesma representação anterior, mas, com $\mathrm{K}$ valendo 7

\begin{tabular}{|l|c|c|c|c|c|}
\hline Câmera & $\begin{array}{c}\text { Médi } \\
\mathbf{a}\end{array}$ & $\begin{array}{c}\text { Median } \\
\mathbf{a}\end{array}$ & $\begin{array}{c}\text { Maio } \\
\mathbf{r}\end{array}$ & $\begin{array}{c}\text { Meno } \\
\mathbf{r}\end{array}$ & $\mathbf{M + m}$ \\
\hline $\begin{array}{l}\text { Samsun } \\
\mathrm{g}\end{array}$ & $63,6 \%$ & $60 \%$ & $\begin{array}{c}90,9 \\
\%\end{array}$ & $32,2 \%$ & $\begin{array}{c}85,4 \\
\%\end{array}$ \\
\hline Canon & $74,5 \%$ & $72,7 \%$ & $29 \%$ & $85,4 \%$ & $\begin{array}{c}54,5 \\
\%\end{array}$ \\
\hline Média & $69 \%$ & $66,3 \%$ & $\begin{array}{c}59,9 \\
\%\end{array}$ & $58,8 \%$ & $\begin{array}{c}69,5 \\
\%\end{array}$ \\
\hline
\end{tabular}

\subsection{BASE DIRIK ET AL. (2008)}

Para testes realizados com a base de dados de Dirik, Sencar e Nemon (2008). O índice de acertos foi de $95 \%$, superando o resultado obtido pelo autor da base, usando filtro com $\mathrm{K}$ de valor 19. Nessa base existiam 2 câmeras e 100 imagens de cada uma. 0 conjunto foi dividido entre 80 imagens de cada dispositivo para treinamento e o restante para o teste. Do total de 40 testes, apenas 2 indicarem um resultado negativo. Porém, nesse experimento foi necessário uma leve alteração no algoritmo (o tamanho do filtro descrito na abordagem proposta foi aumentado), devido ao fato de que na base criada por esse autor a poeira ou falha das câmeras geram impressões de tamanho bem maiores nas suas imagens.

Tabela 3. Resultados de cada uma das métricas

\begin{tabular}{|l|c|c|c|c|c|}
\hline $\begin{array}{c}\text { Câmer } \\
\mathbf{a}\end{array}$ & $\begin{array}{c}\text { Médi } \\
\mathbf{a}\end{array}$ & $\begin{array}{c}\text { Median } \\
\mathbf{a}\end{array}$ & $\begin{array}{c}\text { Maio } \\
\mathbf{r}\end{array}$ & $\begin{array}{c}\text { Meno } \\
\mathbf{r}\end{array}$ & $\mathbf{M}+\mathbf{m}$ \\
\hline Nikon & $90 \%$ & $90 \%$ & $85 \%$ & $65 \%$ & $95 \%$ \\
\hline Canon & $100 \%$ & $100 \%$ & $85 \%$ & $40 \%$ & $70 \%$ \\
\hline Média & $95 \%$ & $95 \%$ & $85 \%$ & $52,5 \%$ & $\begin{array}{c}82,5 \\
\%\end{array}$ \\
\hline
\end{tabular}

\section{CONSIDERAÇÕES FINAIS}

Este artigo apresenta uma nova e inédita abordagem para a identificação da câmera geradora de uma dada imagem; chamado de SCZ.
O método desenvolvido é extremamente simples em comparação com as outras metodologias encontradas na literatura; haja visto que estas fazem uso de filtros complexos e/ou trabalham no domínio da frequência (wavelet, Fourrier e etc).

O SCZ baseia-se exclusivamente em filtros de DPI simples e cálculos estatísticos básicos, como média e mediana.

Tal fato pode ser comprovado pelos resultados obtidos na Seção 4, de modo que superou alguns métodos reportados, inclusive com a mesma base de dados desses.

\section{REFERÊNCIAS}

DIRIK, A. E.; SENCAR, H. T.; NEMON, N. Digital single lens reex camera identification from traces of sensor dust. IEEE Transactions on Information Forensics and Security (TIFS), 2008.

LUKAS, J. F.; GOLJAN, M. Digital camera identification from sensor pattern noise. IEEE Transactions on Information Forensics and Security (TIFS), 2006.

GERADT, Z. J. et al. Methods for identification of images acquired with digital cameras. Enabling Technologies for Law Enforcement and Security, 2001.

KUROSAWA, K.; KUROKI, K.; SAITOH, N. CCD fingerprint method identification of a video camera from videotaped images. In: IEEE International Conference on Image Processing (ICIP). Proceedings... 1999.

ROCHA, A. et al. Vision of the unseen: Current trends and challenges in digital image and video forensic. ACM Computing Surveys (CSUR), 2011.

KHARRAZI, M.; SENCAR, H.; MEMON, N. Blind source camera identification. In: IEEE International Conference on Image Processing (ICIP). Proceedings... 2004.

Li, C-T. Source camera identification using enhanced sensor pattern noise. IEEE Transactions on Information Forensics and Security, 2010. 